\title{
Comparação entre dois instrumentos de avaliação de amplitude de movimento de ombro após Acidente Vascular Encefálico
}

\author{
Comparison between two instruments for evaluation of shoulder range of motion after \\ Brain Stroke
}

\section{Dabrina Gabriela Oliveira ${ }^{1}$ \\ Daiane Marques Ferreira ${ }^{2}$ \\ Gabriela Xavier Santos ${ }^{3}$ \\ Carolina Kosour ${ }^{4}$ \\ D Luciana Maria dos Reis ${ }^{5}$}

Endereço Científico: Universidade Federal de Alfenas (UNIFAL-MG), Campus II - Santa Clara. Av. Jovino Fernandes de Sales, 2600 - Santa Clara, Alfenas - MG, 37133-840

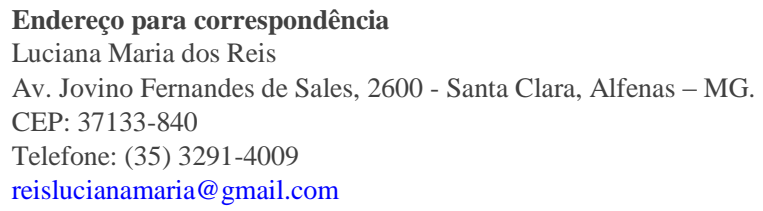

${ }^{1}$ Graduanda do Curso de Fisioterapia da Universidade Federal de Alfenas (UNIFAL-MG)

Alfenas-MG, Brasil.

jesusnidia@gmail.com

${ }^{2}$ Mestranda do Programa de Pós-Graduação em Ciências da Reabilitação da Universidade Federal de Alfenas (UNIFAL-MG) Alfenas-MG, Brasil.

daiane_987@ hotmail.com

${ }^{3}$ Mestranda do Programa de Pós-Graduação em Ciências da Reabilitação da Universidade Federal de Alfenas (UNIFAL-MG) Alfenas-MG, Brasil.

gabi_xaviersantos@yahoo.com.br

${ }^{4}$ Doutora, docente do curso de Fisioterapia da Universidade Federal de Alfenas (UNIFAL-MG)

Alfenas-MG, Brasil.

carolina.kosour@unifal-mg.edu.br

${ }^{5}$ Doutora, docente do curso de Fisioterapia da Universidade Federal de Alfenas (UNIFAL-MG)

Alfenas-MG, Brasil.

reislucianamaria@gmail.com

\section{Resumo}

Introdução: Após o Acidente Vascular Encefálico (AVE), ocorre mudança no tônus muscular que implica em diminuição da amplitude de movimento (ADM) do ombro. O instrumento mais utilizado para medir a ADM é o goniômetro universal, porém, existem outros métodos, como a fotogrametria.

Objetivo: Avaliar a eficácia do Kinovea na avaliação da ADM de flexão e abdução do ombro pós-AVE.

Materiais e métodos: Participaram do estudo 19 voluntários pós-AVE crônico. A avaliação da ADM de flexão e abdução de ombro foi realizada através da goniometria e análises fotográficas pelo Kinovea. As medidas máximas obtidas nas avaliações foram submetidas a verificação de normalidade pelo teste Shapiro Wilk, as variáveis calculadas foram comparadas pela Correlação de Pearson, com um nível de significância $(\alpha)$ de 0,05 .

Resultados: As análises demonstraram correlação alta $(r>0,954 ; p=0,000)$ para flexão de ombro e boa ( $>0,627 ; \mathrm{p}=0,004)$ para abdução de ombro.

Conclusão: O Kinovea apresentou boa eficácia em capturar a ADM de abdução e flexão de ombro nos indivíduos avaliados.

Palavras-chave: Acidente vascular encefálico. Fotogrametria. Goniometria articular. Ombro.

\begin{abstract}
Introduction: After a stroke, there is a change in muscle tone that implies a decrease in the range of motion (ROM) of the shoulder. The most used instrument to measure ROM is the universal goniometer, however, we have other methods, such as photogrammetry.

Objective: To evaluate the effectiveness of Kinovea in the assessment of shoulder flexion and abduction ROM after stroke. Materials and methods: Nineteen chronic post-stroke volunteers participated in the study. The evaluation of the shoulder flexion and abduction ROM was performed through goniometry and photographic analyzes by Kinovea. Statistical analysis, the maximum measures obtained in the evaluations were subjected to verification of normality by the Shapiro Wilk test, the calculated variables were compared by Pearson's Correlation, with a significance level $(\alpha)$ of 0.05 .

Results: The analyzes showed a high correlation $(r>0.954 ; p=0.000)$ for shoulder flexion and good $(r>0.627 ; p=0.004)$ for shoulder abduction.

Conclusion: Kinovea showed a good efficiency in capturing the ROM of abduction and shoulder flexion in the evaluated individuals.
\end{abstract}

Key words: Vascular Brain Accident. Photogrammetry. Goniometry. Shoulder.

\section{Cite como \\ Vancouver}

Oliveira, SG, Ferreira DM, Santos, GX, Kosour, C, Reis, LM. Comparação entre dois instrumentos de avaliação de amplitude de movimento de ombro após Acidente Vascular Encefálico. Conscientiae Saúde 2019 out./dez.;18(4):480-488.

https://doi.org/10.5585/conssaude.v18n4.15381. 


\section{Introdução}

O acidente vascular encefálico (AVE) é decorrente de alteração na vascularização encefálica que pode ser de origem isquêmica, resultante de fenômenos tromboembólicos, ou devido a hemorragia por ruptura de aneurismas ou má formação arteriovenosa. Estas alterações podem ser definitivas ou transitórias na função de uma ou mais áreas do encéfalo ${ }^{1,2}$.

Entre 1990 e 2010, o número de mortes decorrentes do AVE aumentou cerca de $26 \%$ na população mundial, sendo considerado segunda principal causa de morte e principal causa de incapacidade no mundo ${ }^{3,4}$. A presença de comprometimento de linguagem, sensoriomotor e cognitivo, bem como capacidade reduzida para realizar autocuidado e participar de atividades sociais e comunitárias contribuem para a perda de função e presença de incapacidade observadas após o AVE ${ }^{5,6}$.

Dentre os comprometimentos, destaca-se o surgimento de dor no ombro hemiplégico. Esta condição está presente em cerca de $50 \%$ dos sobreviventes e resulta em consequências funcionais, como limitação na função do membro superior e consequente redução da qualidade de vida ${ }^{7}$. A patogênese da dor no ombro hemiplégico é multifatorial podendo ser decorrente de fatores neurológicos como espasticidade e sensibilização central, bem como de fatores mecânicos associados como subluxação do ombro, lesão do manguito rotador, distúrbios da articulação glenoumeral e capsulite adesiva ${ }^{7,8}$.

A fisioterapia exerce importante papel na reabilitação após o AVE, atuando na prevenção e restauração das funções afetadas por meio de um arsenal de técnicas e recursos eletrotermofototerapêuticos com o objetivo de promover melhora da atividade funcional e independência do paciente ${ }^{8,9}$. Um dos objetivos da reabilitação fisioterapêutica no AVE é restaurar ou melhorar a amplitude de movimento (ADM) ${ }^{8,9}$.

O instrumento mais utilizado para medir a ADM é o goniômetro universal, entretanto, sua utilização exige experiência no manuseio pelo terapeuta, para que o risco de erro durante a mensuração seja reduzido ${ }^{10}$. Oferece pouco ou nenhum feedback ao paciente e os resultados são armazenados manualmente, o que torna mais difícil o processamento dos dados obtidos ${ }^{11}$.

Apesar do amplo uso do goniômetro, o avanço da tecnologia tem permitido o desenvolvimento de instrumentos de análise que oferecem avaliação mais precisa, confiável e de baixo custo. O Kinovea é um software de fotogametria que consiste em um sistema de análise de vídeo bidimensional, permitindo a avaliação de vários componentes do movimento como ADM, velocidade, aceleração, tempo e distância da trajetória ${ }^{12,13}$. 
Embora tecnologias com desenvolvimento de softwares venham sendo implementadas por profissionais da saúde ${ }^{14,15,16}$ com o objetivo de avaliar e analisar o progresso da reabilitação, existem poucos estudos na literatura sobre a aplicabilidade do Software Kinovea na mensuração da ADM de ombro, particularmente após o AVE. Por este motivo, fez-se necessária a avaliação da efetividade deste recurso por meio de comparação com um instrumento de avaliação já consagrado na literatura.

\section{Matérias e métodos}

Trata-se de estudo clínico e prospectivo. A pesquisa foi aprovada pelo Comitê de Ética da UNIFAL-MG (CAAE: 58830816.0.0000.5142) e possui cadastro no Registro Brasileiro de Ensaios Clínicos (REBEC: RBR-55smwr), respeitando todas as normas e diretrizes da resolução 466/12 do conselho nacional de saúde (CNS).

As avaliações foram realizadas na Clínica de Fisioterapia da Universidade Federal de Alfenas (UNIFAL-MG), Campus II - Santa Clara e o recrutamento da amostra ocorreu por busca ativa na cidade de Alfenas-MG e região.

Foram incluídos no estudo indivíduos com diagnóstico clínico de AVE em fase crônica (seis meses ou mais), de ambos os sexos, com idade de 20 a 80 anos, movimentos ativos de abdução e flexão de ombro, observados por avaliação prévia, espasticidade menor que dois pela Escala Modificada de Ashworth (EAM) para a musculatura espástica de MMSS, aspectos cognitivos preservados, avaliados pelo Mini Exame de Estado Mental (MEEM), e que estavam de acordo com o Termo de Consentimento Livre e Esclarecido (TCLE). Foram excluídos do estudo indivíduos que apresentavam patologias neurológicas associadas e/ou outras condições dolorosas do ombro. Considerando os critérios de inclusão e exclusão, a amostra final foi constituída por 19 indivíduos.

\section{Avaliação da amplitude de movimento}

\section{Goniometria}

Primeiramente foi realizada a goniometria de abdução de ombro, onde o paciente foi posicionado em ortostatismo, membro superior ao longo do corpo, eixo posicionado próximo ao acrômio, barra fixa sobre a linha axilar posterior e barra móvel acompanhando o movimento de abdução, na região dorsal (Grau $0^{\circ}$ a $180^{\circ}$ ). Em seguida, foi realizada goniometria de flexão de ombro, na qual o paciente foi posicionado em ortostatismo, membro superior ao longo do corpo, eixo posicionado próximo ao acrômio, barra fixa sobre a linha axilar média do tronco 
apontando para o trocanter maior do fêmur e barra móvel sobre a superfície lateral do corpo do úmero voltado para o epicôndilo lateral ${ }^{17}$.

\section{Software Kinovea}

Foram colocadas esferas de isopor de $11 \mathrm{~cm}$ na região do acrômio e epicôndilo lateral ${ }^{18}$ e as imagens foram captadas por um equipamento da marca Xiaomi, modelo Redmi Note 8. O paciente foi posicionado em ortostatismo e orientado a realizar flexão e abdução máximas de ombro de forma ativa. As imagens foram capturadas no início e final da amplitude de movimento e, posteriormente, foram analisadas por meio do software Kinovea, que é um software aberto criado para análise de movimento (Kinovea, 0.8 15, http://www.kinovea.org ) (Figura 1).

Figura 1 - Amplitude de movimento do ombro analisado pelo Software Kinovea. A: abdução ativa do ombro; B: Flexão ativa do ombro

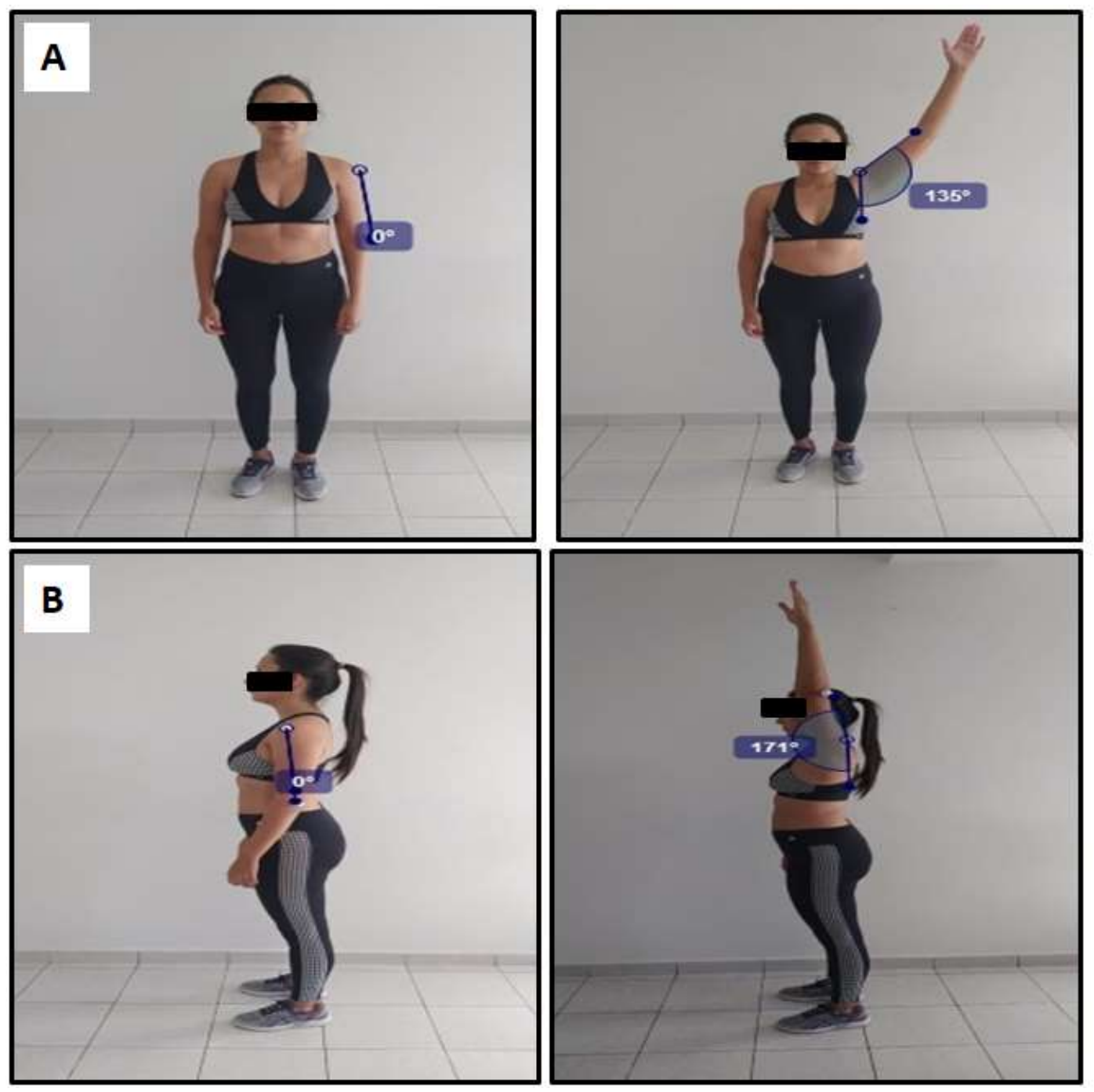

Fonte: Do autor. 


\section{Análise Estatística}

Foi verificada normalidade dos dados pelo teste de aderência de Shapiro Wilk. As variáveis foram comparadas pela Correlação de Pearson para verificar a força da relação entre os instrumentos utilizados. Foram considerados valores de $r$ abaixo de 0,40 como correlação baixa; entre 0,41 e 0,59, moderada; entre 0,60 e 0,79, boa e acima de 0,80, alta. Foram apresentados dados com Média e Erro Padrão da Média (EPM) e considerado nível de significância de 0,05 .

\section{Resultados}

Na tabela 1, são apresentados resultados da Correlação de Pearson entre as medidas obtidas na goniometria e no Software Kinovea, onde é possível observar alta correlação para flexão de ombro e correlação boa para o movimento de abdução.

Tabela 1 - Correlação de Pearson entre os dois métodos utilizados para avaliação

\begin{tabular}{lllll}
\hline Variável & Comparação & R & p- $(<0,05)$ & Correlação \\
\hline & & & & \\
Flexão de ombro & Kinovea/Gonio & 0,954 & 0,000 & Alta \\
Abdução de ombro & Kinovea/Gonio & 0,627 & 0,004 & Boa \\
\hline
\end{tabular}

Legenda- r: correlação de Pearson; p: significância estatística; gonio: goniometria. Fonte: Do autor.

Na tabela 2, são apresentados os valores médios e de EPM das variáveis obtidas por meio da goniometria e do Software Kinovea.

Tabela 2 - Valores de média e erro padrão das análises obtidas com o Software Kinovea e a Goniometria

Médias (Erro Padrão)

\begin{tabular}{lll}
\hline Variável & Kinovea & Goniometria \\
\hline & & \\
Flexão de ombro & $97,05 \pm 9,69$ & $90,74 \pm 9,53$ \\
Abdução de ombro & $82,21 \pm 9,17$ & $98,89 \pm 8,02$ \\
& & \\
\hline Fonte: Do autor. & &
\end{tabular}




\section{Discussão}

Conforme apresentado na literatura, o Kinovea apresenta grande aplicabilidade para calcular ângulos articulares, deslocamentos horizontais, verticais e angulares, velocidades e acelerações, tornando-se uma ferramenta útil para mensuração clínica da ADM, no entanto, existem poucos estudos na literatura sobre a sua aplicabilidade do na mensuração da ADM de ombro, particularmente após o AVE.

Foi realizado um estudo de casos por SANTOS \& NASCIMENTO ${ }^{19}$, no qual utilizaram o Software Kinovea para análise qualitativa da movimentação de flexão e abdução de ombro com duas voluntárias mastectomizadas, uma com cirurgia há menos de um ano e outra há mais de um ano, submetidas a 15 sessões de exercícios baseados na movimentação natural de forma individual. Foram observadas melhoras expressivas na qualidade de vida (QV); amplitude de movimento $(\mathrm{ADM})$ de flexão e abdução $\left(40^{\circ} / 70^{\circ}\right)$ de ombro na voluntária 1 e $\left(10^{\circ} / 10^{\circ}\right)$ na voluntária 2, homolateral à cirurgia; e melhora no desempenho físico.

Outro estudo, realizado por RAMOS \& CATELA $^{20}$, utilizou o Software Kinovea para análise da postura de sentado em bebê hipotônico de 9 meses de idade sob a demanda funcional de alcançar e agarrar brinquedos e mostrou que a estimulação funcional propiciou mudança de padrão de controle postural sentado.

$\mathrm{KRÜGER}^{21}$ analisou as diferenças entre as posições angulares de flexão do ombro durante a execução de batimentos do vôlei: impacto no volante, clear, amortie e remate. Participaram neste estudo 3 atletas com média de idade de 30,67 $\pm 3,06$ anos, onde cada atleta efetuou 10 repetições dos 3 batimentos em análise, enquanto era filmado no plano sagital por duas câmeras Casio exlim que captavam 190 imagens por segundo. As referências anatômicas do lado dominante de cada atleta foram sinalizadas, através de marcadores nas espinhas ilíacas antero e póstero superiores, bordo lateral do acrômio e epicôndilo lateral e medial do cotovelo. Os vídeos dos batimentos foram analisados pelo Kinovea - versão 15. Neste estudo piloto, o clear foi o batimento no qual os atletas contactaram mais cedo o volante, seguido do remate e por último do amortie.

Em outro estudo, realizado por $\operatorname{COSTA}^{22}$ com 11 pacientes crônicos após AVE, foi utilizado ambiente virtual usando o XBOX 360 Kinect ${ }^{\circledR}$, onde os participantes realizavam 15 tentativas no jogo de dardos em uma única sessão e a cinemática do membro superior era registrada em vídeo para análise no Software Kinovea. Concluiu-se que ângulos e velocidades podem ser trabalhados com o uso do jogo de dardos virtual de acordo com o grau de 
comprometimento neurológico, sendo de grande importância para o planejamento da fisioterapia motora dos pacientes com AVE.

Levando em consideração o número de estudos que utilizam o Software Kinovea na avaliação fisioterapêutica e a escassez de estudos que comprovem a efetividade do mesmo na avaliação da ADM após AVE, fez-se necessária a realização desse estudo, uma vez que a mensuração da ADM é de grande importância para reconhecer movimentos deficitários e determinar terapias eficazes, além do fato de que a utilização de Softwares, como o Kinovea, podem favorecer a avaliação de baixo custo de forma mais precisa que a avaliação convencional.

Neste estudo, o Kinovea demonstrou eficácia na avaliação da ADM estática de abdução e flexão de ombro nos indivíduos avaliados em comparação aos dados coletados de goniometria, com pouca variação.

Observou-se, pelos resultados das análises estatísticas, alta correlação para flexão de ombro e correlação boa para o movimento de abdução. Demonstrando que o Kinovea foi capaz permitir avaliação dos movimentos de forma precisa e confiável.

Além disso, o Software Kinovea torna a avaliação mais rápida, o que evita a fadiga muscular e auxilia na avalição de pacientes pós AVE. Como o terapeuta deve estar mais distante do paciente para capturar a fotografia, permite a visualização do mesmo como um todo, tornando mais fácil a identificação de compensações e ajuste do comando verbal responsável pela realização correta do movimento.

Embora tenha sido observada, como limitação do estudo, a dificuldade de alcançar um maior número de indivíduos que se encaixassem nos critérios de inclusão, os resultados do presente estudo comprovaram eficácia do Kinovea na avaliação da amplitude de movimento de abdução e flexão de ombro na amostra estudada.

Os resultados observados, juntamente com o fato de se tratar de um instrumento prático e de baixo custo, sugerem sua utilização na prática clínica e contribuem com informações para novos estudos que incorporem o Software Kinovea na avaliação de disfunções neuromusculoesqueléticas.

\section{Conclusão}

Conclui-se que o Software Kinovea apresentou uma boa eficácia em capturar a ADM de abdução e flexão de ombro nos indivíduos avaliados, com pouca variação dos dados coletados, de maneira rápida, simples, segura e baixo custo. 


\section{Agradecimentos}

Os autores agradecem o apoio financeiro da Fundação de Amparo à Pesquisa do Estado de Minas Gerais (FAPEMIG) e Coordenação de Aperfeiçoamento de Pessoal de Nível Superior (CAPES).

\section{Referências}

1. Pompeu JE, Alonso TH, Masson IB, Pompeu SMA A, Torriani-Pasin C. Os efeitos da realidade virtual na reabilitação do acidente vascular encefálico: Uma revisão sistemática. Motricidade. 2014; 10(4): 111-122.

2. Lopes JM, Sanchis GJB, Medeiros JLAD, Dantas FG. Hospitalização por acidente vascular encefálico isquêmico no Brasil: estudo ecológico sobre possível impacto do Hiperdia. Rev. bras. epidemiol. 2016; 19: 122-134

3. Lozano R, Naghavi M, Foreman K, Lim S, Shibuya K, Aboyans V, et al. Global and regional mortality from 235 causes of death for 20 age groups in 1990 and 2010: a systematic analysis for the Global Burden of Disease Study 2010. The lancet. 2012; 380(9859): 20952128.

4. Hankey GJ. Secondary stroke prevention. Lancet Neurol. 2014; 13(2): 178-194.

5. Faria CD, Silva SM, Corrêa JC, Laurentino GE, Teixeira-Salmela LF. Identification of ICF participation categories in quality-of-life instruments utilized in cerebrovascular accident victims. Rev. panam. Salud pública. 2012; 31(4): 338-344.

6. Rodgers H. Stroke. Handbook of Clinical Neurology. 2013; 110: 427-433.

7. Manara JR, Taylor J, Nixon M. Management of shoulder pain after a cerebrovascular accident or traumatic brain injury. J Shoulder Elbow Surg. 2015; .24(5), 823-829437.

8. Vasudevan JM, Browne BJ. Hemiplegic shoulder pain: an approach to diagnosis and management. Phys Med Rehabil Clin N Am. 2014; 25(2), 411-437

9. Brainin M, Zorowitz RD. Advances in stroke: recovery and rehabilitation. Stroke. 2013; 44(2): 311-313.

10. Ferreira DM, Carvalho LC, Kosour C, Santos ATS, Marin LF, Santos GX, et al. Inter-and intra-rater analysis of hemiparetic shoulder abduction using PhysioPlay ${ }^{\mathrm{TM}}$. Acta Fisiátrica. 2019; 26(3).

11. Arya KN, Pandian S, Vikas, Puri, V. Rehabilitation methods for reducing shoulder subluxation in post-stroke hemiparesis: a systematic review. Top Stroke Rehabil. 2018; 25 (1): $68-81$. 
12. Guzmán-Valdivia CH, Blanco-Ortega A, Oliver-Salazar MA, Carrera-Escobedo JL. Therapeutic motion analysis of lower limbs using Kinovea. Int J Soft Comput Eng. 2013; 3(2): 2231-307.

13. Costa HJB. Análise cinemática de pacientes com acidente vascular cerebral durante jogo de dardos em ambientes virtual e real [dissertação]. Centro de Ciências da Saúde, Universidade Federal do Rio Grande do Norte, Natal, 2017.

14. Krawczky B, Pacheco AG, Mainenti MR. A systematic review of the angular values obtained by computerized photogrammetry in sagittal plane: a proposal for reference values. $\mathbf{J}$ Manipulative Physiol Ther. 2014; 37(4): 269-275.

15. Ribeiro AFM, Bergmann A, Lemos T, Pacheco AG, Russo MM, de Oliveira L AS, et al. Reference values for human posture measurements based on computerized photogrammetry: a systematic review. J Manipulative Physiol Ther. 2017; 40(3): 156-168.

16. Choi KY. Analysis of facial asymmetry. Arch Craniofac Surg. 2015; 16(1): 1.

17. Marques AP. Manual de goniometria. 2a ed. Barueri: Manole, 2003.

18. Bullock MP, Foster NE, Wright C C. Shoulder impingement: the effect of sitting posture on shoulder pain and range of motion. Man Ther. 2005; 10(1): 28-37.

19. dos Santos DM, do Nascimento VC, Peressim LB. Proto movimentação natural: aplicação de protocolo em mulheres mastectomizadas. Revista de Trabalhos Acadêmicos da FAM. 2017; 2(1).

20. Ramos CV, Catela, D. Análise de recorrência da postura de sentado em bebé hipotónico de 9 meses de idade sob constrangimento funcional de alcançar e agarrar brinquedos. Atividade física em populações especiais. 2015; 1: 99-112.

21. Krüger G. Estudo Preliminar das Características Eletromiográficas da Articulação do Ombro no Saque Flat no Tênis: Comparação Entre as Técnicas Foot-back e Foot-up. Monografia (Trabalho de conclusão de curso em Educação Física) Universidade Federal de Santa Catarina, 2014.

22. Costa HJB. Análise cinemática de pacientes com acidente vascular cerebral durante jogo de dardos em ambientes virtual e real [dissertação]. Centro de Ciências da Saúde, Universidade Federal do Rio Grande do Norte, Natal, 2017. 